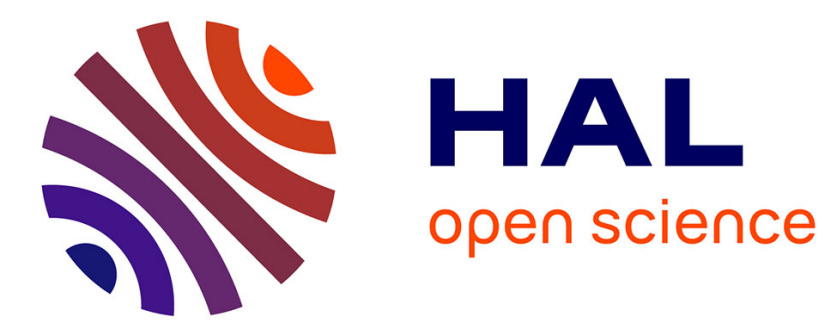

\title{
L'éthique embarquée
}

Nicolas Lechopier

\section{To cite this version:}

Nicolas Lechopier. L'éthique embarquée: Faut-il un éthicien dans une équipe de recherche en santé publique?. Éthique et Santé, 2015, 12 (2), pp.124-129. 10.1016/j.etiqe.2014.12.003 . hal-01100231v2

\section{HAL Id: hal-01100231 \\ https://hal.science/hal-01100231v2}

Submitted on 12 May 2017

HAL is a multi-disciplinary open access archive for the deposit and dissemination of scientific research documents, whether they are published or not. The documents may come from teaching and research institutions in France or abroad, or from public or private research centers.
L'archive ouverte pluridisciplinaire HAL, est destinée au dépôt et à la diffusion de documents scientifiques de niveau recherche, publiés ou non, émanant des établissements d'enseignement et de recherche français ou étrangers, des laboratoires publics ou privés.

\section{(2)(1) $\$$}

Distributed under a Creative Commons Attribution - NonCommercial - ShareAlikel 4.0 


\title{
L'éthique embarquée
}

\section{Faut-il un éthicien dans une équipe de recherche en santé publique ?}

\author{
Nicolas Lechopier
}

Univ Lyon, EA-4148, Faculté de médecine Lyon Est, Université Lyon1, École normale supérieure de Lyon, 38, boulevard Niels-Bohr, 69100 Villeurbanne, France

Version auteur de l'article paru dans Éthique et santé (2015) 12, 124-129

http://dx.doi.org/10.1016/i.etiqe.2014.12.003

\begin{abstract}
Résumé
À quelles conditions un regard «éthique » peut-il échapper à l'instrumentalisation, a fortiori s'il est embarqué dans le champ de pratiques qu'il prend en vue ? Cette question se pose de façon d'autant plus aiguë que l'éthique est couramment incorporée dans les activités soulevant des questions socialement vives (innovations technoscientifiques, guerrières, etc.). Tout en cherchant à développer un regard distancié, d'instruire le débat éthique et social, comment ne pas considérer que des spécialistes de l'éthique - et plus largement les chercheurs des disciplines des sciences humaines et sociales travaillant sur les enjeux éthiques et sociétaux - travaillent en fait à cautionner et vernir les projets controversés pour assurer leur acceptabilité ? De fait, être embarqué c'est faire partie mais aussi prendre parti. À quels genres d'exigences répond le travail de l'éthique ? À qui faut-il rendre des comptes ? L'article fait le point sur ce questionnement en ce qui concerne l'éthique de la recherche en santé publique, particulièrement en santé mondiale. En posant des jalons pour une démarche de recherche en éthique structurée autour d'un travail descriptif, interprétatif et réflexif, cet article s'attache à souligner des pistes de résolution des difficultés de toute éthique embarquée. La question des modalités de reddition de comptes s'avère cruciale.
\end{abstract}

\section{Summary}

Under what conditions can the "ethical" gaze avoid being instrumentalized, and this in particular when looks at the field of practice from within? This question arises even more acutely when ethics is constantly incorporated into activities where social live issues arise (technological and scientific innovation, war technology, etc.). Even when ethical specialists attempt to distance themselves from their topics and to enrich the ethical and social debates, how can one avoid thinking that they - and more generally the researchers in the human and social sciences working on ethical and societal problems - are in fact engaged in legitimizing and rubberstamping controversial projects in order to make them acceptable? In fact, being engaged in a practice is taking part of it, but also being partial to it. What kind of requirements should ethical work live up to? To whom is one accountable? This article takes on this question in the context of public health research ethics, a field still in the making. Laying down the foundations for research into ethics, structured around a descriptive, interpretative and reflexive study, this article highlights some possible ways to resolve the difficulties that any engaged ethics must face. The question of the modalities of accountability is here crucial. 


\section{L'éthique embarquée. Faut-il un éthicien dans une équipe de recherche en santé publique ?}

En mars 2003, des centaines de journalistes accompagnent les militaires américains lors de l'invasion de l'Irak. Depuis navires, bases mobiles et chars, ces journalistes donnent des nouvelles $\mathrm{du}$ front. Professionnels, indépendants ou employés d'organes de presse, ce sont les journalistes embarqués (embedded), incorporés au dispositif militaire. Un débat s'installe d'emblée. Ces journalistes, qui ont suivi une formation spécifique par l'armée américaine avant même le déclenchement de l'intervention [1], doiventils encore être considérés comme des journalistes ou comme des agents de propagande ? Que vaut leur privilège d'être au plus près des points chauds pour rendre compte du conflit, alors que celui-ci ne s'exerce que sous les conditions dictées et prévues par l'un des belligérants ? Le terme «embarqué » désigne le fait d'être «à bord ». Il désigne non seulement la proximité à quelque chose mais aussi la situation d'y être engagé. L'embarquement est une inscription dans un dispositif qui rend possible une activité, mais en la mettant au service de celui-ci. Pour l'embarqué, faire partie c'est aussi probablement prendre parti. Si l'on attend des journalistes un regard indépendant, informé et distancié, leur embarquement est une situation paradoxale : elle leur donne les moyens de réaliser leur travail de journaliste tout en le mettant en péril.

Les éthiciens - si tant est que cette catégorie soit fondée - ont pour rôle d'analyser des situations, de faire usage de référentiels normatifs ou évaluatifs, de contribuer à la reconstruction de ces référentiels, de donner des conseils, d'aider à produire des avis, etc. Leur institutionnalisation constitue en quelque sorte une situation d'embarquement. Pour avoir accès aux cas, pour être au plus près des situations soulevant des questions éthiques, ils risquent de se voir perdre aussitôt ce qu'on peut supposer être l'un des points d'accroche de leur légitimité : une forme d'indépendance et d'autonomie du jugement et un recul critique permettant de regarder cette situation d'un œil décalé. C'est d'ailleurs là la question de la posture de «l'éthicien ». À quels genres d'exigences répond-il ? À qui doit-il rendre des comptes?

\section{L'éthique by design}

Dans sa Théorie du Drone, Chamayou s'attache en particulier à décortiquer le raisonnement d'intellectuels recrutés pour interroger les mutations contemporaines de l'éthique de la guerre, et notamment l'éthique du recours à des drones [2]. Les drones sont des aéronefs téléguidés et armés, utilisés principalement pour des bombardements et des exécutions extra-judiciaires ciblées. Leur mise en action est sans risque physique pour le pilote, puisqu'il est situé à grande distance de l'action. À en suivre ces éthiciens, les drones constituent tout compte fait un progrès moral dans l'art de la guerre, puisqu'ils diminuent la mortalité et permettent des actions plus sécurisées. Tout en démontant leurs arguments fallacieux, Chamayou dénonce cette transformation de l'éthique en «nécroéthique ». Les nécroéthiciens sont en quelque sorte la figure la plus sombre des éthiciens embarqués. 
La figure de l'éthicien devenu complice de projets mortifères se retrouve en filigrane dans toutes les critiques de l'enrôlement. C'est le cas dans les écrits du groupe Pièce et main d'CEuvre qui dénoncent l'enrôlement des sciences humaines sociales dans les processus d'acceptabilité des «nécro-technologies » [3]. De fait, depuis la fin des années 1980, il est devenu courant de faire place à des spécialistes de l'éthique et des enjeux sociétaux dans les projets de recherche en sciences de la vie, en santé, en ingénierie, etc. La décision marquante de ce point de vue est celle consistant à consacrer 3 à $5 \%$ du financement du Projet Génome Humain (HGP, dans les années 1990) à l'étude des « implications éthiques, juridiques et sociales (ELSI) » [4]. Des politiques du même ordre furent proposées dans les années 2000 pour le champ des nanotechnologies, et aujourd'hui dans la biologie de synthèse ${ }^{1}$. À une autre échelle, les financements sur projets de recherche en santé et en environnement encouragent aujourd'hui souvent à inclure des chercheurs pour travailler sur les aspects sociétaux et/ou éthiques.

Nous sommes donc entrés dans une période où sont prises en compte, de plus en plus tôt et par anticipation, les difficultés d'ordre éthique qui pourraient venir interférer avec les trajectoires d'innovations espérées. Par exemple, dans le best-seller de Thaler et Sunstein consacré aux nudges, il est frappant de s'apercevoir à quel point le questionnement éthique est intégré dès la formulation de leur proposition. Les nudges («coups de pouce») sont un outil de changements des comportements individuels issus du marketing social. Ils consistent à modifier l'architecture du choix de telle sorte que les gens soient amenés à adopter le «bon » comportement, du point de vue économique ou du point de vue de la santé. Ce genre de dispositif de manipulation douce a été popularisé par ce livre en l'accompagnement de son prêt-à-penser éthique, le paternalisme libertarien [5]. L'éthique embarquée, ce n'est donc pas seulement l'embarquement des éthiciens, mais c'est une sorte d'éthique « by design », incorporée dans l'outil - donc potentiellement vidée de toute portée critique.

Sous couvert de développer un regard distancié, d'étudier les stratégies de résistance et de détournements, d'instruire le débat éthique et social, comment ne pas considérer que des spécialistes de l'éthique - et plus largement les chercheurs des disciplines des sciences humaines et sociales travaillant sur les enjeux éthiques et sociaux - travaillent en fait à cautionner et vernir les projets controversés pour assurer leur acceptabilité ? À quelles conditions un regard éthique peut-il échapper à l'instrumentalisation, a fortiori s'il est embarqué dans le champ de pratiques qu'il étudie ?

\section{Incorporer l'éthique dans la santé publique}

Voyons ce qui se passe dans les domaines de la santé. Dans le champ de la clinique, à l'hôpital, des spécialistes en éthique ont été, à partir des années 1960 et de façon croissante, intégrés dans les comités de révision de la recherche, les services d'éthique clinique, les modules de formations en

1 L'Institut de biologie synthétique de l'université de Berkeley comprend un programme « E3LSA » (Ethical, Economic, Environmental, Legal \& Social Aspects). À Londres, le Centre for Synthetic Biology and Innovation (CsynBI) est co-dirigé par le sociologue Nikolas Rose. 
éthique, etc. Cette institutionnalisation aurait ainsi contriué à transformer la pratique clinique, elle aurait permis de reconsidérer la place des patients dans la relation médicale et de réhabiliter le « sujet » face à l'avancée de l'objectivation biomédicale.

Dans le champ de la santé publique, c'est semble-t-il avec l'émergence du paradigme de la santé mondiale que les réflexions éthiques ont trouvé une nouvelle institutionnalisation. Le champ de la santé mondiale (Global Health), qui a émergé au tournant des années 2000, s'intéresse aux problématiques de santé qui traversent les frontières (pandémies, styles de vie, migrations, développement, droits, etc.), nécessitent un regard en termes de promotion de la santé [6], concernent directement des déterminants sociaux de la santé [7] et impliquent des coopérations transnationales [8] et interdisciplinaires [9]. Dans les années2000,un certain nombre d'initiatives ont été prises pour développer la présence et le discours de l'éthique dans le champ de la santé publique à l'échelle globale : rapport du Nuffield Council of Bioethics [10], initiatives de l'OMS pour «incorporer l'éthique dans la santé publique» [11], création de revues académiques spécialisées en éthique de la santé publique [12], séminaires sur les valeurs de la santé mondiale, etc. [13]. Traditionnellement, les approches philosophiques de la santé publique s'étaient plutôt consacrées à un travail sur les fondements, réfléchissant sur les valeurs sous-jacentes et la justification des procédures de décision. Le travail critique était plutôt pris en charge par des anthropologues ou des sociologues ayant choisi un regard de distanciation, interrogeant les partispris et les dérives de la santé publique. La structuration d'un champ de recherche en éthique de la santé publique permet de faire travailler ensemble ces deux approches. C'est du reste ce que suggère Deborah Lupton, pour qui le temps d'un rapprochement entre la philosophie de la santé publique et la sociologie critique est venu [14]. Les questions abordées dans ces différentes initiatives portent sur des enjeux éthiques tels que la justice dans la répartition de la santé, l'adéquation des agendas de recherche aux besoins des populations, l'accès au système de santé, la prise en compte des inégalités de santé et de leurs déterminants sociaux, la participation des personnes aux services de santé et aux recherches, l'articulation entre les valeurs d'autonomie et les approches collectives et populationnelles de la santé publique, etc. L'éventail des problématiques éthiques de la santé publique est, on le voit, particulièrement large.

Depuis peu, des acteurs privés comme la fondation Bill \& Melinda Gates, bailleur de fond majeur dans le champ de la santé mondiale, financent des études sur ce genre de questions d'éthique. Les équipes de recherche peuvent susciter la réalisation d'études, non plus seulement sur les implications (comme dans les volets ELSI) mais sur les «enjeux [issues] éthiques, sociaux et culturels $(\mathrm{ESC})$ ». Le curseur, en se déplaçant des «impacts » vers les « enjeux », témoigne de l'incorporation croissante des problématiques d'éthique - en tous cas de celles qui sont identifiées par les chercheurs et les institutions qui les financent - dès le départ. La nouveauté est aussi que ces études s'exercent dans le cadre de consultations pour les acteurs de la recherche, renouvelant ainsi les modes d'interaction entre éthique et recherche en santé. Distinctes des comités d'éthique institutionnels où les spécialistes en éthique trouvent habituellement leur place, ces activités de consultation offrent à des praticiens et des chercheurs un espace dédié pour analyser les enjeux éthiques, sociaux et culturels pendant le montage de leur projet, son déroulement ou ses retombées. Cela permet en quelque sorte de «débureaucratiser » l'éthique en ne la cantonnant pas au seul 
moment de l'examen obligatoire du protocole par un comité, préalable à sa mise en œuvre. Dans les comptes rendus de cette expérience, quelques difficultés sont signalées [15]. Au-delà des difficultés institutionnelles d'articulation avec les comités d'éthique de la recherche et les enjeux de représentativité, les questions posées portent aussi bien sur le positionnement intellectuel adopté dans ces activités que sur les liens d'intérêts et d'indépendance vis-à-vis des équipes de recherches.

Ces difficultés se retrouvent en fait dans d'autres situations où l'éthique est mobilisée aux côtés de la recherche. En Amérique du nord et en Angleterre, plusieurs programmes de recherche en santé mondiale ont inclus dans leurs activités des recherches sur l'éthique. J'ai pour ma part conduit un projet de recherche en éthique au sein d'un projet financé par l'Initiative de recherche en santé mondiale du Canada [15]. Cette recherche m'a permis notamment de développer des études de cas invitant à questionner les formes de responsabilités des chercheurs en santé mondiale [16]. J'ai également pu montrer que dans certains cas les tensions éthiques rencontrées dans l'application ou la restitution des résultats renvoyaient à des options scientifiques et axiologiques plus fondamentales [17]. D'autres chercheuses en éthique de l'université de Brock en Ontario travaillant dans le même espace institutionnel, et accompagnant également les chercheurs sur leur terrain de recherche, ont conduit des enquêtes du même genre, interrogeant en particulier la nature des partenariats de recherche impliquant des circulations entre Nord et Sud ainsi que les problèmes liés à l'hétérogénéité des évaluations éthiques selon les régions et les institutions [18].

De telles recherches en éthique sont articulées à des projets de recherche qu'elles prennent pour objet de questionnement. Elles y sont d'une certaine façon embarquées. Quelle est donc la juste posture pour ce genre de recherches en éthique ? Quel angle de recherche adopter, et pour quelles conséquences dans la relation avec les institutions de recherche et des chercheurs? Dans la suite de ce texte, je proposerai des éléments de construction d'une posture de recherche en éthique qui, sans résoudre tous ces problèmes, permet de comprendre où ils se posent.

\section{Faire de la recherche en éthique}

Considérons les questions éthiques, non pas comme renvoyant d'emblée à des énoncés de jugements de valeurs, mais bien plutôt comme ce qui suppose une certaine sorte d'expérience. Lors de la formulation des questions de recherche, du recueil de données, des analyses, etc., les participants à un projet de recherche en santé publique - les personnes «cibles », les chercheurs, les proches, témoins, etc. - font à ces occasions différentes expériences, éprouvent des émotions, s'engagent dans certaines formes d'interactions sociales, etc. Ces expériences - incorporées, vécues mais pas forcément de façon individuelle ou privée - peuvent dans certains contextes - réunions, protestations, recueil de consentement, enquête etc. - faire l'objet d'une reprise réflexive et d'énonciations différentes. Ces énonciations à leur tour peuvent être articulées entre elles pour tisser le fil d'un questionnement éthique.

Une recherche en éthique consiste à documenter ces expériences, leurs mises en mots et les interactions qui les accompagnent. Il s'agit par exemple de s'intéresser aux processus de reconnaissance entre partenaires, à la justesse d'une attitude au carrefour de plusieurs exigences parfois contradictoires, aux façons dont des risques définis à distance lors de la rédaction d'un 
protocole sont requalifiés par les personnes concernées, aux motivations des personnes ou des chercheurs à agir d'une certaine façon dans un contexte particulier, aux enjeux de sincérité et de respect dans les dialogues interculturels, etc. Tous ces phénomènes engagent, pour parler comme Bernard Williams des «concepts épais » [19]. S'ils peuvent assurément être définis et clarifiés, ces concepts entrelacent des faits et des valeurs. Pour accéder à cette épaisseur, il faut mobiliser des instruments de recherche qualitative adéquats - observations, entretiens individuels et collectifs, recueil de récits, etc.

Ces outils permettent par exemple de décrire comment ce qui était prévu dans un projet de recherche se transforme en pratique, au contact du terrain, suivant toute une série d'épreuves et d'ajustements qui révèlent la distance observable entre le prescrit et le réel et qui s'avèrent signifiants d'un point de vue éthique. L'enquête qualitative peut s'intéresser également aux questions éthiques émergentes et imprévues, par exemple les controverses sur le partage des responsabilités, les «aides ancillaires » et tous les enjeux soulevés par les relations don/contre-don qui caractérisent la relation de recherche [16]. Elle peut aussi interroger les processus de redéfinition des identités qui ont lieu dans les controverses éthiques [20] ou la constitution d'une diversité d'expériences morales [21].

Cette tâche de mise en lumière et de description des situations et expériences morales se double d'un travail interprétatif et compréhensif qui dépend en bonne partie des référentiels disciplinaires mobilisés. Sans entrer dans le détail ici, on peut souligner la variété de ces référentiels. Certains mobiliseront des outils de bioéthique appuyés sur des principes [22], d'autres utiliseront une réflexion sur l'interculturalité [23], d'autres encore se poseront dans le cadre interprétatif de «l'éthique comme pratique» [24] ou de l'éthique de la discussion impliquant «experts » et «profanes » [25]. La philosophie des sciences est elle aussi en mesure de fournir des modèles qui permettent de situer les stratégies et les interactions des acteurs des recherches en santé comme des façons d'articuler des valeurs éthiques et épistémiques. Par exemple, il n'est pas rare que les recherches donnent lieu à des controverses portant sur la question suivante : faut-il suivre les «bonnes pratiques » de recherche qui consistent à réaliser le protocole tel qu'il a été conçu afin de sauvegarder la validité de ses résultats attendus, ou bien l'adapter aux suggestions des personnes concernées ou aux nouveaux paramètres pertinents surgissant lors de la réalisation du terrain afin de renforcer la pertinence contextuelle ? Pour interpréter et clarifier ce genre de difficultés, le modèle développé par Hugh Lacey peut être utile [26,27]. Plus largement, les travaux issus des «études de sciences » permettent de recontextualiser sur le plan épistémologique, éthique et sociologique ce genre de questions.

Au-delà de ce travail descriptif et interprétatif, la recherche en éthique consiste enfin à nourrir une pratique réflexive. Sur le terrain, le questionnement sur l'éthique peut en effet se constituer comme un espace de réflexivité et de retour critique sur les valeurs directrices de l'activité et sur leur réalisation en pratique. Des outils d'animation et de formation relevant de l'analyse de la pratique, de l'auto-confrontation croisée, la mise en place de forums locaux permettent d'ouvrir un tel espace de réflexion. Pour cela, certaines conditions doivent être réunies. Il faut que les partie-prenantes de la recherche non seulement aient le souhait de prendre en compte les questions d'éthique mais se donnent le temps de le faire. Le phénomène de l'accélération globale n'aide pas à libérer du temps 
disponible pour la réflexion éthique [28]. «L'éthicien » est-elle d'ailleurs peut-être le nom de cet îlot de temps libre pour explorer les enjeux éthiques, délibérer à propos des valeurs sous-jacentes, à propos de la responsabilité, de la juste posture ou des effets de domination.

En résumé, la recherche en éthique se déploie selon trois sortes d'activités : description de l'observable, processus d'interprétation et outil de réflexivité. Cette conception de la recherche en éthique permet-elle de répondre aux difficultés de l'enrôlement soulignées plus haut, dans quelle mesure et à quelles conditions ?

\section{L'éthique et la reddition de comptes}

Il faut bien reconnaître que cette conception d'une recherche éthique n'échappe pas davantage que les postures «d'éthique embarquée » précédemment évoquées aux difficultés de la situation d'embarquement. D'abord, pour mener des observations et documenter les situations, il faut bien aller «sur place ». Cela suppose toujours dans une certaine mesure de passer des compromis et de donner des gages aux interlocuteurs, y compris avec ceux qui possèdent le plus de pouvoir. De plus, l'activité réflexive n'a de sens que si elle se produit dans un espace accessible aux praticiens et mobilisable par eux. Comme partie de l'enquête en éthique, elle présuppose elle aussi une certaine forme d'engagement sur le terrain auprès des acteurs, d'une façon qui ne peut d'ailleurs pas être tout-à-fait détachée des enjeux de reconnaissance et de rapports de force.

La recherche en éthique est donc «embarquée» aussi bien en raison de sa démarche observationnelle que par sa dimension interprétative et réflexive. Comme on le sait, le commanditaire, qu'il soit institutionnel ou individuel, a une certaine importance dans les directions prises par des recherches, dans le choix des paramètres pris en comptes, dans les outils d'analyse mobilisés, etc. De plus, le chercheur pour être accepté et reconnu par les acteurs locaux doit fournir un certain nombre de gages et de garanties. Faire une recherche en éthique telle que décrite plus haut implique d'être inséré dans des multiples relations d'influence et de pouvoir, dans des institutions qui ne peuvent pas ne pas influer sur le travail de recherche. C'est bien là le problème de la situation d'embarquement : la difficulté pour une telle recherche en éthique n'est peut-être pas tant une question de posture, ni même le fait d'être ou non embarquée sur le terrain, mais peut être bien de déterminer à qui elle doit rendre des comptes de son activité.

En matière de recherche en santé publique, il existe des acteurs minoritaires : ceux qui a priori ne sont pas en mesure d'influencer l'agenda de la recherche ou ses partis-pris. Ce sont généralement les participants et/ou les personnes qui sont d'ailleurs aussi souvent les plus concerné(e)s par ses résultats, ou encore ceux et celles qui au contraire ne participent pas à un projet donné parce qu'ils ne satisfont pas aux critères d'inclusion par exemple. L'asymétrie entre ces acteurs «mineurs » (comme les participants, les habitants) et «majeurs », dotés de pouvoir (comme les chercheurs, les financeurs) n'est bien entendu pas écrite dans la nature des choses. Elle n'est pas non plus une structuration intrinsèque de toute recherche scientifique ou de santé publique. Elle est le fruit d'un processus dynamique : Deleuze et Guattari montrent bien comment les minorités se définissent, non pas de façon quantitative, mais bien par un processus de devenir-minoritaire [29]. Or justement les écrits et les catégories de l'éthique de la recherche, qui ont le souci de tenir compte des minorités en 
les qualifiant en fonction du fait qu'elles font l'objet d'une attention particulière (par exemple les « populations vulnérables »), tendent en même temps à les figer et les enfermer dans cette positionlà.

L'autre piste, sur laquelle pourrait s'engager la recherche en éthique, consisterait au contraire à « rendre des comptes » à ceux à qui la situation d'embarquement ne nécessite pas d'en rendre. Il s'agirait de conduire la recherche en éthique de sorte à être en mesure de l'assumer devant toutes les personnes qui n'ont justement dans cette recherche pas le plus de pouvoir, voire aucun. Cette exigence de reddition de comptes est difficile à satisfaire dans la mesure où l'on trouvera souvent peu d'appui institutionnel pour le faire (de la même façon qu'en général les recherches en population ne font guère l'objet de restitutions aux personnes les plus concernées). La difficulté est également épistémologique. Comme les chercheurs en sciences sociales le savent, ces activités de restitution et de reddition de compte posent souvent de redoutables difficultés [30]. Dans quelle mesure doivent-elles reprendre à leur compte les catégories des acteurs et les catégories normatives existantes ? Une restitution concernant les aspects éthiques peut-elle parler de la même façon aux acteurs majoritaires et minoritaires ? Ces questions se posent, en effet. Et d'ailleurs, s'il est si difficile de concevoir ce que serait un «spécialiste en éthique ", c'est peut-être que cette matière, qui peut faire l'objet d'une investigation sensible, interprétative et critique, déborde inévitablement les postures d'expertise. L'éthique embarquée, c'est donc une éthique qui doit assumer le choix d'un bord.

\section{Références}

[1] Bizimana AJ. Le dispositif embedding: surveillance et intégration des journalistes en Irak. Québec: Presses de l'université du Québec; 2014, 215 p.

[2] Chamayou G. Théorie du drone. Paris: La Fabrique; 2013, 363 pages.

[3] Pièces et main d'œuvre. Aujourd'hui le nanomonde \#19 - Biologie de synthèse etacceptabilité. 2012.

[4] ELSI Research Planning and Evaluation Group. A review and analysis of the ELSI research programs at the National Institutes of Health and the Department of Energy. National Human Genome Research Institute; 2000, 57 pages.

[5] Thaler RH, Sunstein CR. Nudge : la méthode douce pour inspirer la bonne décision. Paris: Vuibert; 2012, 469 pages.

[6] Deschamps J-P. Une « relecture » de la charte d'Ottawa. Sante Publique 2003;15:313-25.

[7] Commission des Déterminants Sociaux de la Santé. Closing the gap in a generation: health equity through action on the social determinants of health. Geneva: World Health Organization; 2008.

[8] Beaglehole R, Bonita R. What is global health? Glob Health Action 2010;3, 10.3402/gha.v3i0.5142.

[9] Koplan JP, Bond TC, Merson MH, et al. Towards a common definition of global health. Lancet 2009;373:1993-5.

[10] Nuffield Council on Bioethics. Public health: ethical issues. London: Nuffield Council on Bioethics; 2007, 225 pages.

[11] Coleman CH, Bouësseau M-C, Reis A. The contribution of ethics to public health. Bull World Health Organ 2008;86:578A.

[12] Dawson A, Verweij M. Public health ethics: a manifesto. Public Health Ethics 2008;1:1-2.

[13] Benatar SR, Lister G, Thacker SC. Values in global health governance. Glob Public Health 2010;5:143-53. 
[14] Lupton D. Some thoughts on public health ethics; 2012. Simplysociology.wordpress.com, [accédé le 12/01/2014].

[15] Stephen C, Daibes I. Defining features of the practice of global health research: an examination of 14 global health research teams. Glob Health Action 2010;3:5188.

[16] Lechopier N. Éthique et justice dans les recherches participatives ancrées dans les communautés. Le cas d'une enquête écosystémique en Amazonie. Ethique Publique 2011;12:20123.

[17] Lechopier N. Modèles conceptuels en santé-environnement et éthique de la restitution des résultats. Une étude de cas. Environ Risques Sante 2013;12:250-7.

[18] McGinn M, Tilley SA. Research ethics on the ground: partnerships, practices, and plans in global population health - a collective case study of ethics issues and challenges. Global Social justice and the Social Determinants of Health: Setting the Course for the Future; 2012, 7 pages. [19] Williams B. L'éthique et les limites de la philosophie. Paris: Gallimard; 1990, 243 pages. [20] Laurent B. Les politiques des nanotechnologies : pour un traitement démocratique d'une science émergente. Paris: Charles Léopold Meyer; 2010, 242 pages.

[21] Hunt MR, Carnevale FA. Moral experience: a framework for bioethics research. J Med Ethics 2011;37(11):658-62.

[22] Beauchamp TL, Childress JF. Les principes de l'éthique biomédicale. Paris: Les Belles Lettres; 2008, 645 pages.

[23] Stewart K, Sewankambo N. Okukkera Ng'omuzungu (lost in translation): understanding the social value of global health research for HIV/AIDS research participants in Uganda. Glob Public Health 2010;5:164-80.

[24] Fassin D. L'éthique, au-delà de la règle : réflexions autour d'une enquête ethnographique sur les pratiques de soins en Afrique du Sud. Societes Contemp 2008;71:117, http://dx.doi.org/10.3917/soco.071.0117

[25] Massé R. Expertises éthiques savantes et profanes en santé publique : défis et enjeux pour une éthique de la discussion. Sante Publique 2012;24:49-61.

[26] Lacey H. Is science value free? Values and scientific understanding. London et New-York: Routledge; 2005, 285 pages.

[27] Lechopier N. Sciences, valeurs et pluralisme chez Hugh Lacey. In: Pierron JP, Parizeau MH, editors. Repenser la nature : dialogue philosophique, Europe, Asie, Amériques. Québec: Presses de l’Université Laval; 2012. p. 61-78.

[28] Rosa H. Aliénation et accélération : vers une théorie critique de la modernité tardive. Paris: La Découverte; 2012, 152 pages.

[29] Deleuze G, Guattari F. Mille plateaux. Capitalisme et schizophrénie. Paris, France: les Ed. de minuit; 2002, 645 pages.

[30] Laurens S, Neyrat F. Collectif. Enquêter : de quel droit? Menaces sur l'enquête en sciences sociales. Éditions du Croquant; 2010, 320 pages. 